larger areas of tissue. Tumour immunology is covered in depth, and almost half the book is devoted to immunotherapy. The description of the experimental basis for this therapy is logical, critical and provides the best and most balanced overview of this subject that I have read. The trials and tribulations of the more recent attempts at immunotherapy of human cancer are precisely reported in the last chapter. This cannot be faulted as a review but I was disappointed by the last section "Present position and future prospects," which is vague and indecisive. Sadly, I suppose this reflects the state of the subject, for if the facts permitted a grand synthesis, surely Professor Woodruff would be the man to make it.

\section{P. Alexander}

Transplantation Today Volume 6. A. P. Monaco, M. L. Wood \& F. T. RAPaPoRT. (1981) New York: Grune and Stratton. 1305 pp. $\$ 37.50$ net.

The main impediment to successful tissue transplantation is immunological rejection. In the case of marrow transplants it is the recipient who may elicit an immune response in the marrow cells of the donor, leading to graft-versus-host disease. The main objectives of current transplantation research are to avoid inducing rejection, either by carefully matching donor and recipient HLA antigens, or by the use of immuno-suppressive drugs. "Transplantation Today" Volume 6 is a collection of papers delivered at the Eighth International Congress of the Transplantation Society, held in Boston, U.S.A. in 1980, concerned largely with the conceptual and practical problems presented by rejection. Because histocompatibility research is continuously defining more tissue antigens however, it may never be possible to achieve the goal of donor-recipient matching. Some workers, believe that this may not be necessary, since few antigens may be crucial in triggering rejection. This explains the relatively large number of contributions relating to the HLA-D/DR antigen system. To the clinician charged with coping with the daily problem of rejection, it is often necessary to use more drastic means to modify immunity, achieved by a selection of generally highly effective immuno-suppressive drugs. However, the long-term use of these drugs has its penalities. One of these is the higher incidence of malignant disease in transplant recipients than in normal individuals. Penn suggests that the incidence of non-Hodgkin's lymphoma is 45-100-fold higher than in the general population. Clearly, these alarming figures have to be set against the potential benefits which accrue from transplantation. It is a subject that might have warranted more space in this volume. The diversity of histocompatibility research generated by finding that the genes coding for transplantation antigens are involved in, or associated with the control of immunity has created something of a dilemma that clearly emerges in this volume.

In attempting to present the current status of transplantation research is it necessary to cover the much wider field of Immunobiology? In addition, many papers are not presenting new results, though they are short enough in most cases for this not to matter too much. But the weight of numbers obscures more fundamental contributions. There is a good argument for reducing many of the papers to abstract lengths.

G. M. TAYLOR

\section{Hodgkin's Disease and the Lymphomas} Vol. 4. Ed. C. R. TAYLOR. (1981) Edinburgh: Churchill Livingstone. 377 pp. $£ 24.00$ net.

This is the 4th Annual Review of Hodgkin's disease and Lymphomas by Clive Taylor, and as usual with his reviews, they are a pleasure to read, with perhaps more attention than might have been expected to making the English intelligible and enjoyable.

It is perhaps inevitable that this review should concentrate mainly on non-Hodgkin's lymphoma, and in particular, on the considerable turmoil in the histological classification of non-Hodgkin's lymphoma which exists at the moment. In this respect, the book fulfils an important and timely role in summarizing and gathering together the wealth of information which has recently been published about the pathological and immunological classification of lymphomas. Dr Taylor and his co-authors wisely do not come to any definite conclusions on which classification is superior, though, because the book has been written and compiled in California at Professor Luke's centre, con- 
siderable attention is paid to the LukesCollins classification. The new international formulation, which is yet to be published, is also mentioned, though of course it cannot be dealt with in any detail at this stage. It will be interesting to see whether, by the time of the next Annual Review, a consenus has emerged that can be reported by Dr Taylor.

The rest of the Review of the literature in the book is thorough, and includes chapters on extra-nodal lymphoma, epidemiological studies of lymphoma and genetic and kinetic studies of both Hodgkin's disease and nonHodgkin's lymphoma. The references which have been accumulated, not only from the English literature but also from the foreign language literature, are exhaustive and very thorough.

In summary this is a book which can be recommended most strongly. It is both enjoyable to read and extremely informative. G. BLACKLEDGE 